\title{
Aportaciones críticas y despatologizantes en la evaluación psicológica de la diversidad de género ${ }^{1}$
}

\author{
Critical and depathologising inputs on psychological \\ assessment of gender diversity
}

KONSTANTINOS ARGYRIOU² (Instituto de Filosofía, CSIC)

Artículo recibido: 7 de marzo de 2021

Solicitud de revisión: 15 de abril de 2021

Artículo aceptado: 31 de enero de 2021

Argyriou, Konstantinos (2022). Aportaciones críticas y despatologizantes en la evaluación psicológica de la diversidad de género. Recerca. Revista de Pensament i Anàlisi, 27(1), pp. 1-24. doi: http://dx.doi.org/10.6035/recerca.5798

\section{Resumen}

El énfasis de la psicología en la vivencia individual ha generado prototipos idealizados sobre la identidad de género, promulgados a través de las herramientas tradicionales de evaluación psicológica. Las personas transgénero y género no-conforme se han visto presionadas a encajar en las concepciones binarias de dichas evaluaciones, hasta la reciente despatologización aprobada por la Organización Mundial de la Salud. En el presente trabajo teórico se examina cómo se fueron transformando los discursos sobre la diversidad de género y la corporalidad en psicología. Para ello, se apela a cuatro argumentos: la movilización activista contra las definiciones homogéneas de lo trans, la aplicabilidad de las categorías de los sistemas taxonómicos, las resistencias contra las escalas de validez de los test psicométricos y, por último, cuestiones de adaptación intercultural de términos, mediciones y concepciones del cuerpo y la identidad de género.

Palabras clave: evaluación psicológica, asesoramiento, identidad de género, diversidad, adaptación cultural.

\footnotetext{
${ }^{1}$ Esta investigación forma parte del contrato FPU17/03809 del Ministerio de Universidades y se enmarca dentro de las actividades del proyecto Epistemologías híbridas: cuerpos, biometrías y ensamblajes (EPHYCUBE, PID2019-105428RB-I00) AEI/10.13039/501100011033 (MCIU/AEI/FEDER, UE) (IP: Eulalia Pérez Sedeño). Mi agradecimiento a Eulalia Pérez Sedeño y a Dau García Dauder por sus indicaciones, a los miembros del proyecto EPHYCUBE por sus comentarios y a las personas que han revisado el manuscrito por haberlo enriquecido tanto.

${ }^{2}$ konstantinos.argyriou@cchs.csic.es
} 


\begin{abstract}
Psychology's emphasis on individual experience has created idealised prototypes for gender identity, promulgated through traditional tools of psychological evaluation. Transgender and gender nonconforming people have been urged to fit into binary conceptions of such evaluations, at least until the recent depathologisation approved by the World Health Organization. The present theoretical study examines how psychological discourses around gender diversity and corporeality have been transformed. To this effect, it appeals to four arguments: to the activist mobilisation against homogeneous definitions of transness, to the applicability of taxonomic systems' categories, to the resistances against psychometric tests' validity scales, and lastly, to issues concerning cross-cultural adaptation of terms, measurements and conceptions of bodily and gender identity.
\end{abstract}

Key Words: psychological evaluation, counseling, gender identity, diversity, cultural adaptation.

\title{
INTRODUCCIÓN
}

La comprensión de las personas trans dentro de la psicología se ha basado históricamente en la evaluación psicológica (o diagnóstico) de la disforia de género, la patologización de la identidad de género y los procesos estrechamente vinculados con la transición corporal - la hormonación y las intervenciones quirúrgicas-. Esa comprensión, enfocada en lo individual, ha omitido demandas de carácter psicosocial, cuestiones que constituyen la personalidad de forma más holística y menos dependiente de la identidad de género, y parámetros intersubjetivos de la relación psicoterapéutica (Bullock y Wood, 2016; Lev, 2004; Richards et al., 2016; Riggle, Scales, McCants y Pascale-Hague, 2011).

La revisión progresiva de las violencias producidas por la psicología ha conllevado un cambio de óptica en la noción de la evaluación en sí: las personas trans y no conformes al género (transgender \& gender non-conforming people, TGNC) ya no se consideran trastornadas, sino personas cuya identidad y expresión de género requiere afirmación y protección frente a vulneraciones y discriminaciones (Shulman et al., 2017). Eso implica que la evaluación gire más en torno a un acompañamiento a procesos que a pautas estandarizadas de detección de comorbilidades.

Por tanto, por evaluación no se entiende aquí solamente la detección de la disforia, como en modelos institucionales, sino también la medición de la angustia, la resiliencia frente al estrés minoritario, el bienestar personal, la calidad de vida y otros factores psicosociales. También se ha de tener en cuenta 
que los dispositivos de dicha evaluación aún no cuentan con una adaptación cultural extendida, sino que provienen del ámbito anglosajón, lo cual genera múltiples campos de acción, algunos plenamente afirmativos y otros todavía anclados en el intervencionismo y el paternalismo.

De forma similar, el uso de test tradicionales de masculinidad y feminidad, más estandarizados en términos de presunta validez y fiabilidad psicométrica, ha mantenido hasta hace relativamente poco una prevalencia llamativa en el caso de las personas TGNC (Miach, Berah, Butcher y Rouse, 200o). Estos test han sido utilizados en casos de detección de lo que se cristalizó como trastorno de identidad de género en el DSM-IV-R (American Psychiatric Association, 2000) y disforia de género en el DSM-5 (American Psychiatric Association, 2013). ${ }^{3}$ Sin embargo, han ido perfilando cierto(s) ideal(es) de subjetividad y corporalidad trans atado(s) al canon y se han mostrado poco sensibilizados en términos de inclusión de personas que no experimentan disforia genital o que tienen expresiones de género alternativas (Davy, 2015; Johnson, 2016).

Además, existen asimetrías, tanto entre la constante creación de nuevas herramientas y la lenta adaptación a ellas por parte de les profesionales de salud mental, ${ }^{4}$ como en la ausencia de un consenso y lenguaje común por parte de los diversos colectivos de psicólogues y terapeutas (Geisinger, 1994; Hambleton y Kanjee, 1995). Eso propicia que la formación continua y la integración de la perspectiva de género en la consulta psicológica devengan tareas difíciles (Bullock y Wood, 2016). Salvo por técnicas muy expandidas que tienen un carácter transversal, como por ejemplo la entrevista clínica o el historial de familia, otras, más específicas, dependen altamente de cada contexto, de la perspectiva de trabajo o del enfoque psicoterapéutico que se aplique. En la última década, la lucha por la despatologización trans y la psicología afirmativa han mostrado un nuevo camino por seguir, eliminando en gran parte las evaluaciones y promoviendo una visión de la intervención psicológica crítica contra los dictámenes históricos restringidos y suspicaces (Singh y dickey, 2017; Suess Schwend, 2020). Un postulado principal del enfoque afirmativo es que la evaluación, particularmente en su faceta institucional asociada a los

\footnotetext{
${ }^{3}$ En realidad, la larga trayectoria de correspondencia entre herramientas diagnósticas y manuales estadísticos de trastornos mentales va mucho más allá del relativamente reciente DSM-IV. No es el propósito de la presente reflexión hacer una genealogía de dicha complicidad. Para una revisión detallada, véase Drescher (2010).

${ }^{4}$ Se utiliza la $e$ a lo largo del texto como forma genérica estratégica de referirse a todos los posibles géneros, no como más correcta, sino como una de las posibilidades de hacer el lenguaje más inclusivo. Se reconoce, sin embargo, que dicho sufijo no está aceptado por la Real Academia Española para un uso extendido. De todas maneras, se desea evitar el uso del masculino como genérico, debido a los sesgos de invisibilización y opresión que el genérico masculino supone.
} 
informes, o cuando se realiza como procedimiento automatizado, contribuye en la perpetuación de una dinámica asimétrica que deshumaniza y objetualiza nuevamente a personas que acuden a la consulta ya heridas. Por ello, se orienta hacia la sustitución de la psicometría por el acompañamiento y la competencia cultural (de la Hermosa Lorenci, 2018; Singh y dickey, 2017).

Paralelamente, la adaptación cultural y lingüística de las herramientas tradicionales, asunto más psicométrico que clínico, ha resultado una tarea complicada de ejecutar debido a las diversas atribuciones y representaciones sobre el género, la sexualidad, el cuerpo y la salud mental en diferentes contextos nacionales y socioeconómicos (Jarrín, 2016). En el caso español, por ejemplo, en cada comunidad autónoma existen aún distintos reglamentos respecto a la atención sanitaria de las personas TGNC. Las comunidades que no disponen de unidades de identidad de género (UIG) redirigen a las personas al sector privado o a otras comunidades (Atienza Macías y Armaza Armaza, 2014; Gómez-Gil et al., 2020), con todas las disparidades que esto pueda generar.

Asimismo, la propia funcionalidad de las UIG y de sus equipos multidisciplinares está atravesada por paradigmas biomédicos de la transición, aunque han ido perdiendo su supuesta legitimidad de diagnóstico progresivamente por los cambios taxonómicos y legislativos (Gómez-Gil et al., 2020). La escucha atenta, la capacitación y el apoyo emocional, el énfasis en el estigma y en la discriminación y el respeto a la particularidad de las experiencias han sido retos más bien secundarios en las dos últimas décadas, mientras el uso de test conservó un carácter tipológico y pegado a las clasificaciones prevalentes. Consecuentemente, la prevalencia del modelo biomédico ha obstaculizado en gran medida la obtención y la comparación de datos cuantitativos y cualitativos.

Colectivos activistas de las últimas décadas han ido abordando críticas y proponiendo prácticas alternativas en el ámbito de la alianza terapéutica y la evaluación psicológica. Asimismo, aunque la última revisión de los estándares de cuidado (SOC) de la WPATH (Coleman et al., 2012) fue decisivamente contraria a la tradición de guardianismo de puerta (gatekeeping), ${ }^{5}$ no consiguió anticipar los cambios significativos en el acompañamiento trans a lo largo de la década (Gregori Flor, García Dauder y Hurtado, 2018). A la espera de la nueva revisión de los SOC, algunas prácticas se han acercado a la autodeterminación y

\footnotetext{
${ }^{5}$ Término que se usa para describir el paternalismo y los requisitos por cumplir para acceder a ciertos tipos de tratamiento.
} 
hacia un modelo de autocuidados que respete las corporalidades y sus particularidades.

Con el fin de visibilizar un aspecto de la práctica psicológica del que se conoce todavía poco a nivel metanarrativo, la presente reflexión teórica procura detallar cuáles han sido las aportaciones a favor y en contra de la evaluación con personas TGNC y sus respectivos lugares de enunciación. Además, con el fin de ofrecer una visión intercultural, se indagará en las razones por las cuales los enfoques tradicionales de patologización e intervencionismo se vieron ralentizados por problemas de adaptación de los instrumentos, pero también por cuestiones institucionales de organización y ofrecimiento de servicios. Para ello, se examinarán:

- los discursos institucionales hegemónicos a favor de las intervenciones corporales y del verdadero transexualismo, en oposición a los discursos periféricos que se alejan del paradigma patologizante,

- las codificaciones de las taxonomías imperantes,

- la resistencia frente a la prueba psicológica, materializada en el malingering, es decir, en la lógica de fingir tener disforia o exagerarla,

- la adaptación sociocultural y lingüística de términos y pruebas que entienden el cuerpo y la identidad como entidades homogéneas, occidentalizadas y medibles.

Analizando esos cuatro parámetros, se desea destacar que los servicios psicológicos para personas TGNC se tienen que alejar del paradigma biomédico y de definiciones restringidas de la corporalidad. El asesoramiento psicológico ya cuenta con una larga historia de estigmatización contra la diversidad de género, de modo que necesita una reconceptualización crítica y una revisión a fondo de sus premisas para asumir la influencia de factores socioculturales en el malestar personal y para liberar los servicios de sus pretensiones paternalistas. La participación ciudadana y la interdisciplinariedad son dos ejes fundamentales de actuación hacia esta dirección. Además, es necesario aprender de las propias personas TGNC y no binarias en dinámicas horizontales, donde ellas sean capaces de sugerir y aprovechar lo que mejor les convenga dentro de la consulta. 


\section{DEFINICIONES Y PARTICULARIDADES}

A lo largo de esta última década, la noción del cuerpo equivocado, a la que han ido apelando tanto discursos biomédicos dominantes como incluso algunos grupos de apoyo trans, ha empezado a debilitarse, gracias a lecturas etnometodológicas y transfeministas que han operado desde los márgenes (Davy, 2015; Hines, 2007; Missé, 2018; Sennott, 2010). Dichas lecturas y políticas de reconocimiento han ido subrayando la importancia de ver a las personas trans ya no como receptores pasivos sino como agentes activos y participativos de una realidad que, hasta hace unas décadas, se construía a su pesar y sin tenerlas en cuenta.

No han sido solo esas voces las responsables de dicho giro, aunque sí las principales. Todo un debate interdisciplinar entre humanidades, ciencias sociales y ciencias de la salud ha permitido el gradual deslizamiento de modelos sociológicos y biomédicos binarios a prácticas y concepciones alternativas de las experiencias de no-conformidad al género, desde lo lingüístico, lo somático y lo político (Vidal-Ortiz, 2008). Paralelamente, y dado que la capacitación de les profesionales de salud mental para asumir las novedades y ofrecer servicios más adecuados es un proceso lento y gradual, que involucra un cambio de actitudes, han ido proliferando servicios - mayormente gratuitos- de redes de contactos y amistades, grupos de apoyo y asociaciones con el propósito de rellenar esa deficiencia (Gregori Flor, García Dauder y Hurtado, 2018). Frente al sistema de complacencia de los discursos biomédicos, la participación ciudadana trazó otro modelo de actuación. En vez de tener que aprender de memoria una performance concreta de género, con manierismos, vestimentas, respuestas preparadas frente a las escalas de masculinidad y feminidad o las entrevistas clínicas y con toda la carga de violencia y patologización que eso conllevaba, la nueva dinámica que empezaron a diseminar las organizaciones trans y LGBTQI era mucho más liberadora.

Por otro lado, el parcial reconocimiento jurisprudencial y legislativo alcanzado por los enfoques afirmativos no refleja una aceptación en los ámbitos biomédico y social (Páez, Hevia, Pesci y Rabbia, 2015). Las actitudes de profesionales de salud mental, de interinos y de estudiantes de ciencias de la salud se miden cuantitativamente (véase Ali, Fleisher y Erickson, 2015), obviando las cuestiones de comunicación y contacto diario, las representaciones psíquicas y sociales que se reproducen en los equipos multidisciplinares y las estrategias particulares de cada centro, clínica y contexto social. La falta de instrumentos de revisión entre profesionales es igual de preocupante (López-Sáez, García 
Dauder, Montero y Lecuona, 2021). A su vez, las reglas del passing, de la medida en que las personas TGNC aceptan los ideales y las normas sobre masculinidad y feminidad, varían según las circunstancias y son relativamente subjetivas y difícilmente medibles en términos pragmáticos (Billard, 2019). La vigilancia de los cuerpos por parte de los sistemas sociales, médicos y legislativos también depende altamente de las interlocuciones de dichos sistemas con los activismos locales y del lenguaje que prevalece en las prácticas clínicas (Kreukels et al., 2010).

La denominada prueba de la vida real es un método de vigilancia que no ha recibido suficiente atención por parte de la academia, de las instituciones, ni siquiera del activismo. Eso se debe a que las exigencias implicadas no siguen un modelo común en todos los países y dependen de los distintos sistemas sanitarios y distribuciones económicas, como se habló anteriormente. Esto puede conducir a la arbitrariedad en la definición de criterios sobre passing y al mayor sufrimiento de las personas afectadas por esos ordenamientos. Dentro de la Unión Europea tampoco existen pautas acordadas, hecho que obliga a muchas personas a migrar a países con mejor atención para conseguir un reconocimiento de su identidad y, por ende, de su cuerpo (European Commission, 2020).

Las críticas respecto a la prueba de la vida real, sin embargo, se restringen por el mismo hecho de que no es fácil acceder a datos cuantitativos y cualitativos. El fallo conceptual intrínseco a dicha prueba, de todas maneras, es que se limita a una lectura visual y superficial de la presentación física, reforzando la conformidad detrás del passing como estrategia de no-descubrimiento, en vez de entender lo trans en toda su riqueza y complejidad, fuera de estereotipos y clichés de masculinidad y feminidad (López-Sáez y García Dauder, 202o). Señalar a lo trans como decepcionante y epistémicamente delimitado ayuda a salvaguardar la estructura cisheteronormativa (Billard, 2019; Fricker y Jenkins, 2017; Johnson, 2016). Esto tiene como resultado, primero, un aumento de presión social y de exigencia para cumplir con los prototipos y, luego, en casos de violencia, por ejemplo, un riesgo de respaldar el acoso contra personas TGNC a través de la estafa y la necesidad de protegerse ante la amenaza de su supuesta falsedad. Dicho de otra manera, si reducimos la legitimidad de las identidades TGNC al éxito del passing, asumimos que la identidad y la corporalidad cis (es decir, no trans) es normativamente mejor y que todo intento de imitarla está destinado a fracasar. Asimismo, sometemos a personas a un sufrimiento psíquico enorme, prescribiendo determinados ideales dañinos de corporalidad (San Emeterio, 2020). 
El giro afirmativo arraigado en el activismo y en la psicología crítica consiste en sustituir las categorizaciones normativas del género, que sirven para perpetuar las injusticias sociales, por definiciones operacionales, abiertas y flexibles, bajo el lema de «primero no dañar (más)» (Singh y dickey, 2017). En la misma línea, descubre otras vivencias de no-conformidad provenientes de ámbitos locales, quizá incluso ininteligibles por parte del establishment biomédico (Jarrín, 2016). Gracias a ese descubrimiento, la afirmación es capaz de revelar las heterogeneidades y las relaciones de poder dentro del propio colectivo TGNC y legitimar cuerpos que normalmente quedarían completamente alejados del campo de visión social.

Promoviendo esta necesidad de situar cada cuerpo dentro de su entorno, Martin y Finn (2010) asocian la autodefinición con las implicaciones concretas que tienen la masculinidad y la feminidad en cada contexto sociocultural. Cuanto más heteronormativo el entorno, más distancia de poder hay entre una persona cis y una persona trans que se autodefinen de la misma manera (por ejemplo, como mujeres). La revisión de las categorizaciones binarias no tiene como objetivo denegar la composición histórica del sistema sexo/género de forma dicotómica, sino examinar cómo esa insistencia ha discriminado epistémicamente a personas que no entran dentro de esos cánones (Fricker y Jenkins, 2017; Johnson, 2016).

Los enfoques afirmativos empiezan por perfilar esta distancia y por cuestionar las presuposiciones normativas que atan a las personas cis al lado de lo inteligible y a las personas TGNC al lado del engaño, la confusión y la incredibilidad. Asimismo, se alejan del discurso de la autenticidad (true transsexualism o discourse of the true self), que incluso desde antes de los años noventa ha procurado modificar relatos personales, para encauzarlos hacia una división cuerpo-mente, cuerpo equivocado-verdadero yo, preop-postop. ${ }^{6}$ Esta idea de reconfiguración de los relatos con el propósito de eliminar contradicciones internas, de localizar la disforia en la infancia y de forzar historias de travestismo infantil descubierto en el acto ha sido objeto de análisis exhaustivos (Mason-Schrock, 1996; Mason-Schrock, Holden y Reid, 2004).7 La equivalencia

\footnotetext{
${ }^{6}$ De esa manera se distingue en la bibliografía médica entre personas que no han recibido una intervención quirúrgica todavía y personas que han complementado los procesos de modificación genital (características primarias del sexo) y corporal (características secundarias).

${ }^{7}$ Algunos de los términos usados, como travestismo, corresponden al contexto sociohistórico de las obras citadas y no se aceptan con la misma facilidad en la actualidad, porque se consideran inadecuados, particularmente desde discursos transfeministas. En otras ocasiones, también se reapropian por parte de los colectivos. Para más información, véase Jarrín (2016).
} 
entre el discurso de la autenticidad y la prueba de la vida real (prácticas de passing) es la que certifica la forma supuestamentecorrecta de vivir la transexualidad.

\section{TAXONOMÍAS}

Aunque es imprescindible tener referencias ampliamente compartidas como la CIE, los SOC o el DSM, justamente para evitar malas prácticas en los servicios psicológicos, la agrupación y la codificación de la diversidad de género en ellas puede provocar malentendidos. Como se ha visto en el apartado anterior, solo algunes de les profesionales de salud mental que trabajan con personas TGNC están concienciades respecto a los aspectos contextuales de las categorías y las definiciones, dado que las formaciones oficiales no hacen especial hincapié en la diversidad de género.

Mientras tanto, ideales universalistas detrás de las clasificaciones y las técnicas de medición borran particularidades culturales. Álvaro Jarrín (2016) describe el caso característico de las travestis en Brasil que, por no ser cómplices con el sistema de medicalización de cuerpos transexuales que dicta el sistema público de salud y las clínicas hospitalarias, quedan fuera de la atención sanitaria y psicosocial. Las normas del passing en la sociedad brasileña premian la intervención quirúrgica y borran, simultáneamente, a quienes se niegan a modificar sus cuerpos y a someterse a discursos patologizantes.

En mayo de 2019, la OMS oficialmente quitó la transexualidad (códigos F64.O-F64.4) de la categoría de trastornos mentales de la undécima versión de la CIE, para colocarla bajo el nombre «incongruencia de género» en otra categoría, la de asuntos relacionados con la salud sexual (HA6o/17-HA61/17) (Castro-Peraza et al., 2019; World Health Organization, 2019). El grupo de trabajo barajaba varias opciones durante años, entre otras quitar la categoría por completo, crear un capítulo especial para ella o incluirla en los diagnósticos de condiciones puramente médicas (Drescher, Cohen-Kettenis y Winter, 2012).

No obstante, no fueron pocas las voces que destacaron que depsiquiatrización no equivale necesariamente a despatologización (Missé, 2018). La incongruencia de género ha pasado a ser una condición de salud en vez de enfermedad o trastorno; sin embargo, se siguen considerando trastornadas las personas intersex, de forma que la estructura biomédica sigue manteniendo su postura paternalista (Castro-Peraza et al., 2019). En el mismo sentido, es distinto entender por DSD un trastorno (disorders of sexual development) y una dife- 
rencia del desarrollo sexual (difference/variation of sexual development) (Gregori Flor, García Dauder y Hurtado, 2018).

No es inusual que les profesionales de salud mental no estén adecuadamente capacitades o atentes a los cambios de los manuales para poder asesorar las disparidades de las experiencias de las comunidades TGNC. Recurrir a la supervisión y la asesoría de otres psicólogues no es siempre una opción y, a veces, se podría poner en cuestión incluso la propia capacitación de les supervisores y sus enfoques de trabajo. Si les usuaries evidencian carencias de competencias culturales en cuanto a los servicios psicológicos ofrecidos, la situación no puede diferir mucho en el caso de los servicios de asesoría profesional. Sin embargo, existe muy escasa bibliografía esclarecedora que detalle la incorporación de las incesantes alteraciones taxonómicas y legislativas o los mecanismos de contratransferencia vigentes por parte de les supervisores (de la Hermosa Lorenci, 2018; Nichols, 2019). ${ }^{8}$

La carencia de habilidades y capacitación adecuadas se encuentra no solo en la práctica clínica, sino también en la construcción y la traducción de herramientas de evaluación. Los riesgos que subyacen clasificando, diagnosticando y etiquetando a las personas TGNC a menudo se ignoran o no se toman en serio. Eso tiene como resultado una estigmatización secundaria o latente, lo que en la literatura aparece como microagresiones (Sue, 2010) (pero que, como término, a lo mejor no refleja los niveles de impacto reales). Las microagresiones en el caso de los test involucran la hipermasculinización o hiperfeminización del contenido de los ítems, la puntuación y la interpretación de los resultados basadas en el sexo asignado al nacer (López Sáez y García Dauder, 2020), pero también el uso de géneros gramaticales incorrectos y expresiones ofensivas, la traducción deficiente de ítems de lenguas que no disponen de género neutro a otras que sí tienen y viceversa. La lucha contra las microagresiones coincide, pues, con la promoción de un lenguaje inclusivo.

\section{DETECCIÓN DE MALINGERING}

Test psicométricos como el MMPI-2, el BSRI y el MCMI, pero también proyectivos, como el Rorschach o el TAT, que son los principales que han sido utiliza-

\footnotetext{
${ }^{8}$ La contratransferencia es un término psicodinámico que señala las proyecciones y los deseos que les asesores inconscientemente plantean a les asesorades, como respuesta a la transferencia recibida, es decir, a la sustitución de patrones de relaciones sustanciales por la relación terapéutica.
} 
dos en el pasado para la evaluación de la disforia de género y la psicopatología en personas TGNC (Affatati, Grattagliano, Todarello y Catanesi, 2012; Krishnamurthy, 2016; Martin y Finn, 2010), disponen de sistemas de interpretación que pueden caer en las microagresiones mencionadas. Esos sistemas se alinean con las taxonomías del DSM y de la CIE, para anteponer los cambios corporales dentro de los márgenes establecidos. Es importante, en ese sentido, entender cuál ha sido la reacción general de las personas sometidas a esos procesos y por qué.

Las escalas de validez en la mayoría de esas evaluaciones tienen la función principal de detectar mentiras. Esta es una de las razones por las que se administran los test, para la revelación de secretos, defensas o conflictos internos que no se hayan detectado en la sesión clínica (Paulson et al., 2019). Esas mentiras pueden ser tanto inconscientes como volitivas. En el caso de las primeras, se habla de ganancia primaria, un alivio inmediato de la tensión que puede suponer un conflicto interno; el mecanismo subyacente en síntomas de conversión. En el segundo caso, se hace referencia a una ganancia secundaria, con beneficios materiales o psicológicos, que se denomina malingering (la idea de fingir una patología intencionadamente) (Domino y Domino, 20o6; Slick y Sherman, 2012).

Dos mecanismos que implican malingering son el exceso de respuestas (overreporting) o la ausencia de respuesta (haber dejado un ítem sin contestar o underreporting). El primero de los dos demuestra conformidad y complacencia, necesidad de convencer a les examinadores de una buena voluntad pero de forma forzada; el segundo demuestra evasión, miedo o rebelión frente a las instrucciones. Pero también se observa otro fenómeno de underreporting, que es el esfuerzo por responder mal, dando muy bajas puntuaciones y reaccionando a las condiciones de la prueba de forma extrema (Slick y Sherman, 2012). Históricamente, este último caso no ha sido muy frecuente en las evaluaciones de personalidad con personas TGNC, justamente porque el propósito principal ha sido complacer a les examinadores para conseguir la aprobación de la hormonación o la operación quirúrgica.

Asimismo, un test verbal puede tener distinta validez de contenido en distintas culturas o grupos sociales. Los test verbales están expuestos a problemas de aculturación, es decir, de imitación de términos y cálculos considerados universales, sin que lo sean (Domino y Domino, 2006). Esos términos y cálculos se esfuerzan por verse reflejados en el DSM; de hecho, dependen de los cambios del DSM para adaptarse adecuadamente y sacar nuevos manuales de instrucciones (Shulman et al., 2017). Pero el DSM refleja las taxonomías del 
sistema sanitario estadounidense, que en casos como este mantiene la disforia como eje principal del diagnóstico, sin asumir además que no se trata de un síntoma exclusivo de las personas trans, o que no todas las personas trans la manifiestan. En el futuro, los manuales de los test es probable que se orienten más hacia la CIE que hacia el DSM (Hogan, 2015), teniendo también en cuenta las modificaciones introducidas en la CIE-11. ${ }^{9}$ De todos modos, es imprescindible que, en vez de acelerar los cambios desarrollando nuevos productos, haya también una fase de asimilación y reposo, para que la investigación ilustre de forma eficaz y crítica sus resultados.

Los test más nuevos, que miden la expresión de género, la disforia y la calidad de vida de las personas TGNC son también portadores de una cultura y una historia específicas. Shulman et al. (2017) realizaron una revisión de ocho de esas herramientas específicas. Evidenciaron que su fiabilidad, información comparativa y generalización eran mínimas, que su sensibilidad al cambio era muy alta y que escalas de validez propiamente dichas no se introducían por parte de los equipos creadores. No obstante, destacan que

El cambio de medidas diagnósticas históricas a herramientas enfocadas en mecanismos específicos que afectan el funcionamiento psicológico como el impacto de la discriminación, la conexión con la comunidad, las estrategias de afrontamiento, y la reflexión acerca de la identidad de género es un paso positivo hacia la afirmación TGNC en la investigación y la práctica psicológicas (Shulman et al., 2017: 11; traducción propia).

Las técnicas proyectivas se supone que están menos sesgadas en términos interculturales, ya que su estructura consiste en la compleción de patrones, en tareas de clasificación o de percepción, y suelen depender menos del lenguaje y más de la intuición o de la ambigüedad perceptiva (Basu, 2014). Se han usado más en pruebas cognitivas que en la medición de la calidad de vida o de masculinidad y feminidad, pero en casos de psicopatología asociada son más eficaces en la detección de mecanismos de defensa sin requerir análisis psicométricos computacionales. Hay quienes defienden que, aún así, los sesgos que se supone que se evitan por vía lingüística en realidad se introducen a través de las imágenes, es decir, por sesgos simbólicos (Hambleton y Kanjee, 1995).

\footnotetext{
${ }^{9}$ El conflicto sobre si la disforia debe o no aparecer en un manual de trastornos psíquicos como el DSM ha incitado un largo debate sobre el cubrimiento de tratamientos por las carteras de servicio. No obstante, eso obscurece el debate real concerniente a la autoridad de dichos manuales o las relaciones de poder entre ellos.
} 
La reproducción de sesgos en el ámbito de los test, teniendo en cuenta sus mecanismos intrínsecos de vigilancia (malingering, controles de passing, experiencia de la vida real), se asocia también a la frecuente ausencia de datos preliminares derivados de administración a poblaciones TGNC. Agrupar lo TGNC dentro de lo LGBQ sin administrarles siquiera los test y, por consiguiente, sin adaptar los ítems adecuadamente ha tenido serias consecuencias epistémicas (Bullock y Wood, 2016; Miach, Berah, Butcher y Rouse, 200o). En ese sentido, es grato recordar que la escala MF (5) del MMPI se usó inicialmente para la detección de hombres gay, naturalizando la masculinidad y la feminidad como opuestas (Krishnamurthy, 2016; López Sáez y García Dauder, 2020), además de que, durante muchas décadas, hasta bien llegados los 2000, orientación sexoafectiva e identidad de género se veían sistemáticamente confluidas (Davy, 2015; Drescher, 2010) a través de la concepción, por ejemplo, de las mujeres trans como hombres gay hiperafeminados y de los hombres trans como mujeres lesbianas hipermasculinizadas.

\section{ADAPTACIÓN SOCIAL, CULTURAL Y LINGÜÍSTICA}

Un cuarto aspecto por examinar es la adaptación del proceso evaluador a diversos ámbitos socioculturales. Tres factores a tener en cuenta cuando se realiza una adaptación social, cultural o lingüística de una herramienta psicológica son las diferencias contextuales respecto a formatos, las cuestiones técnicas y los modos de interpretación (Hogan, 2015). Por ejemplo, un formato de selección múltiple puede suponer desventajas, no solamente cognitivas sino también procesuales, para una población que está más acostumbrada a preguntas abiertas. Del mismo modo, la traducción de un término a otro idioma (por ejemplo, cis, TGNC, genderqueer, entre otros) es posible que no tenga la misma equivalencia o reconocimiento social y eso afecta directamente a la validez de contenido.

Dentro del marco del asesoramiento psicológico, cuestiones relacionadas con la diversidad de género son más específicas que otras, lo cual requiere a personas competentes en materia (Carroll y Gilroy, 2002; Lev, 2004). Encontrar a gente bilingüe o experta en otros idiomas que maneje terminologías y vocabularios inclusivos deviene una tarea ardua, en ese sentido. Es posible que aquelles profesionales que participan en la elaboración y la adaptación de herramientas relacionadas con la identidad de género estén más sensibilizades respecto al uso del lenguaje y la contextualización cultural desde el principio 
(Francia-Martínez, Esteban y Lespier, 2017). No obstante, tanto los equipos de trabajo de elaboración y modificación de herramientas como los de revisión de taxonomías, siguen líneas estandarizadas que se alejan de los postulados de abogacía de colectivos LGBTQI (Drescher, Cohen-Kettenis y Winter, 2012).

A su vez, profesionales que no están capacitades para trabajar con poblaciones TGNC tienen que saber mínimamente a quién redirigir la demanda (Lev, 2004). Un problema similar surge si tienen que emplear herramientas de trabajo para el acompañamiento. La aportación de los activismos puede ser esclarecedora en cuanto a la modificación de los manuales de uso, las prácticas de buena administración e interpretación y la lucha contra la discriminación secundaria o implícita desempeñada por los test. Ya que ni siquiera se suele ofrecer formación especial sobre el malingering (Paulson et al., 2019), los test deben ser herramientas de consulta e información complementaria, enriquecedoras para el acompañamiento al facilitar la cercanía y la transferencia (Basu, 2014), no detectores de mentira, ni determinantes de buenas o malas personas trans. Dicho de otra manera, es pertinente que las pruebas cambien de connotación y, en vez de servir como mecanismos de control o de comprobación, se establezcan como formas de exploración y autoconocimiento a fondo.

En cuanto al proceso de estandarización, no se pueden repetir fácilmente las condiciones de las pruebas piloto de las herramientas, ni encontrar muestras semejantes con facilidad. Si la primera validación no ofrece las evidencias esperadas, las futuras adaptaciones quedan en suspenso y no se renueva la información sobre la validez (Shulman et al., 2017). Además, asumir que todas las personas TGNC, independientemente de su contexto social, apuestan por una coherencia entre la identidad interiorizada y la expresión de dicha identidad hacia fuera implica repetir los mismos sesgos que sirven para fijar las identidades en la cisheteronormatividad y en una presunta universalidad. De todas maneras, a nivel epistémico la transición es ya por definición un proceso mutante y fluido y, por tanto, no cumple con los criterios psicométricos y estadísticos que requieren las herramientas de atención psicosocial para validarse.

La adaptación es también propensa a una serie de factores: primero, las muestras, ya limitadas numéricamente, no sirven para estudios longitudinales. Muchas personas TGNC se desvinculan de los servicios cuando termina su transición o no muestran la misma motivación que en las primeras fases una vez han alcanzado los resultados corporales deseados. Asimismo, las muestras suelen estar sesgadas, incluyendo solo a personas que se dirigen a servicios psicológicos o que desean transitar corporalmente. Personas críticas hacia la 
práctica psicológica o alejadas de los cánones de masculinidad y feminidad no se ven representadas en ellas. Por último, la bibliografía que ofrece líneas sobre la adaptación de los test está muy esparcida por revistas y manuales, no concierne en la mayoría de los casos temas de género y sigue enfoques psicométricos de muy diversa índole.

Otra cuestión que se ignora dentro del marco de la adaptación de paradigmas es el nivel de familiarización de cada cultura con la diversidad de género. Regresando a la argumentación de Jarrín (2016), la institucionalización de las intervenciones corporales y de los sistemas de evaluación por las clínicas de género estadounidenses seguramente se asocie con los ideales de individualismo y de passing de dicha sociedad. Su exportación, siguiendo las imposiciones neoimperialistas, a culturas más colectivistas y lejanas mediante las taxonomías puede cubrir manifestaciones locales que no necesiten encasillar lo TGNC o que operen de distintas maneras.

En ese sentido, pueden variar también los niveles de normatividad social, las exigencias de la prueba de vida real y el conformismo de les profesionales de salud mental con las normas, los test y las taxonomías binarias (Elischberger, Glazier, Hill y Verduzco-Baker, 2018; Monro, 2007). Sabemos, por ejemplo, que el modelo de la terapia triádica, de la cultura de la comprobación y de las unidades de género proviene de Estados Unidos de mediados del siglo pasado, y que los ideales que promueve dan por sentados muchos presupuestos sobre el género (Drescher, Cohen-Kettenis y Winter, 2012; Lev, 2004). El enfoque afirmativo tampoco tiene las mismas repercusiones fuera de los contextos angloamericanos en los que se engendró y es necesario tener en cuenta que, cuanto más transexcluyente y reticente es el contexto sociocultural, mayor será la dificultad de afirmar una identidad TGNC para que luego ella sea insertada en dicho entorno hostil. Dicho de otra manera, si los términos difundidos por los movimientos anglosajones no tienen contrapartidas directas en otros contextos (véase Ho, 2021; Jarrín, 2016), la aplicación de la afirmación como práctica puede encontrar resistencias serias.

La diferencia entre adaptación cultural y competencias culturales es que la primera suele hacer referencia a la contextualización y la traducción de una práctica o una herramienta psicológica a otro contexto cultural (normalmente, a otro país u otra lengua), mientras que las segundas se desarrollan en relación con diferentes colectivos sociales, pero pueden estar dentro de un mismo contexto cultural. Aún bajo estas circunstancias, las dos nociones comparten una idea subyacente de ajuste, localización y distancia de poder. La adaptación de un conocimiento de una realidad sociocultural a otra tiene un valor trans- 
formador $\mathrm{y}$, por consiguiente, una responsabilidad respecto a qué cuerpos legitima y qué cuerpos descarta de los márgenes que establece (Wilkinson, 2014). También por ello, quizá la adaptación de pruebas y cuestionarios, sin contar con las perspectivas émicas de las poblaciones trans locales, y los pasos hacia la despatologización que se llevan a cabo por parte de cada sociedad acarreen más obstáculos epistémicos que oportunidades.

\section{CONCLUSIONES}

Apoyadas en la idea de que las diferencias culturales, económicas y legislativas entre países alteran las manifestaciones conductuales de lo trans, Isabel Esteva y Esther Gómez-Gil (2013) parecían sostener, en las antípodas del discurso de la autenticidad, que la asesoría psicológica no debería ser obligatoria para personas que desean transitar quirúrgicamente, sino dependiente de las necesidades individuales de cada usuarie. A esto añadían que las clasificaciones internacionales son bastante genéricas como para contener todas las particularidades corporales y emocionales de personas diagnosticables con disforia. El diagnóstico, según ellas, se basa exclusivamente en información proporcionada por las personas y la gran diversidad de relatos, vivencias y situaciones es prácticamente insostenible en términos universales. Aunque esta visión y lo desarrollado a lo largo de este artículo coinciden plenamente, en la práctica el gatekeeping promovido por las UIG ha seguido un camino más restrictivo a lo largo de la década (Castro-Peraza et al., 2019; Missé, 2018; Suess Schwend, 2020).

Después del análisis en esos cuatro ejes presentados, se hace patente que la integración de una perspectiva concienciada frente a las particularidades de las personas trans y género no-conforme en los servicios psicológicos es crucial para mejorar sus experiencias y sus interacciones con les profesionales. La oferta de cursos de especialización y el cambio en los planes de estudio universitarios mejoran considerablemente dichas interacciones.

No obstante, reducir la solución del problema solo a asuntos educativos conlleva el peligro de la simplificación. La homogeneización de las experiencias corre el riesgo de perder la oportunidad de examinarlas en su complejidad y particularidad. Tanto el contexto terapéutico como la evaluación psicológica tienen como premisa intrínseca recurrir a un conocimiento existente, a unos estándares preestablecidos y a las referencias de personas que ya han tenido similares experiencias, de un lado o de otro. 
No hay que perder de vista, sin embargo, que la relación terapéutica o el proceso de acompañamiento es un lugar de experimentación también para les propies practicantes de la psicología. Un diálogo situado con les clientes, desde la vulnerabilidad y la codependencia, es una oportunidad valiosa para cuestionar sesgos propios, deconstruir el binarismo desde posiciones interseccionales y crear modos de ver y alianzas que puedan servir para romper con la posible desconfianza y resistencia o con la carga histórica que tiene la disciplina. Lynne Carroll y Paula Gilroy (2002) han ofrecido una serie de recomendaciones sobre la capacitación cultural que nos pueden ayudar a conceptualizar mejor esa confianza en la particularidad de cada experiencia. Entre otras, destacan la exploración del desarrollo de la identidad de género en términos históricos y sociopolíticos, aparte de psicológicos; la discusión de nuevas terminologías; la comunicación abierta de sentimientos de curiosidad voyerista y de cuestiones de contratransferencia; el respaldo de posicionamientos no-binarios y noconformes; la conexión con asociaciones y el apoyo por parte de supervisores especializades; la transformación del contexto de vida y no de la personalidad individual a costo de la adaptación y, por último, la aproximación a enfoques terapéuticos narrativos y constructivistas. El último fin es convencer a les profesionales de que, en palabras de Castro-Peraza et al. (2019: 980; traducción propia), «el trauma psicológico y el sufrimiento no son inherentes a las personas trans pero son el resultado del fracaso de la sociedad en adoptar la diversidad corporal».

El papel del activismo transfeminista ha sido y sigue siendo fundamental en cuanto a la reivindicación de espacios de protección y apoyo, donde primen la honestidad, la espontaneidad y la empatía. La histórica distancia de poder en la interacción entre psicólogues y usuaries ha dado paso a la toma de decisiones mutua, activa y participativa solo en las últimas dos décadas y solo en contextos que han permitido un cuestionamiento mínimo de las distinciones binarias. Como se ha descrito, el binomio autonomía-paternalismo a veces deviene falso, puesto que grupos de apoyo han intentado en ocasiones ocupar una posición paternalista y porque, al otro extremo, existen ya evaluaciones y herramientas afirmativas que ofrecen apoyo sin patologizar y enfatizan en la autodeterminación.

El presente texto opta abiertamente por la necesidad de que sigan disponibles los servicios psicológicos para personas TGNC y por que les psicólogues se apoyen en conocimientos clínicos; no obstante, no se deberían usar dichos conocimientos sino con fines de debatir la transfobia interiorizada, asesorar a las personas en sus procesos y acompañar desde el cuidado, desde un consen- 
timiento informado sustancial y no impregnado por el miedo al arrepentimiento o a las posibles represalias. Asimismo, es precisa una adaptación crítica y émic de herramientas, manuales y prácticas profesionales, siempre y cuando sean de aprovechamiento para les propies solicitantes. Asumiendo las particularidades de cada sistema sanitario, de cada contexto lingüístico y de cada comunidad dentro de lo TGNC, se disminuyen las posibilidades de caer en la trampa de volver a categorizar, estigmatizar o llegar a una homogeneización de las distintas experiencias.

La presente reflexión no ha pretendido ser exhaustiva. El giro despatologizador de la CIE-11 se hace oficialmente vigente del 2022 en adelante y su reflejo en las prácticas cotidianas de los sistemas sanitarios tardará aún más en aparecer, dadas las variaciones legislativas. El conocimiento generado al respecto está en constante transformación y su utilidad se restringe a la idea de que el acompañamiento psicológico puede ser de utilidad para las personas TGNC. Se requieren más investigaciones críticas sobre la relación entre práctica psicológica y diversidad de género y más voces desde distintos contextos, lenguas y cuerpos.

\section{BIBLIOGRAFÍA}

Affatati, Valeria, Grattagliano, Ignazio, Todarello, Orlando y Catanesi, Roberto (2012). Utilizzo del test di Roschach nella diagnosi di disturb di identitá di genere e nella valutazione dell' idoneità alla surgical sex-reassignment. Rivista Di Psichiatria, 47(4), 1-8. doi: https://doi.org/10.1708/1139.12562

Ali, Nareesa, Fleisher, William y Erickson, Julie (2015). Psychiatrists' and psychiatry residents' attitudes toward transgender people. Academic Psychiatry, 40(2), 268-273. doi: https://doi.org/10.007/s40596-015o308-y

American Psychiatric Association (2000). Diagnostic and statistical manual of mental disorders (4th ed., text revision). Washington, DC: American Psychiatric Association.

American Psychiatric Association (2013). Diagnostic and statistical manual of mental disorders (5th ed.). Washington, DC: American Psychiatric Association. 
Atienza Macías, Elena y Armaza Armaza, Emilio José (2014). La transexualidad: Aspectos jurídico-sanitarios en el ordenamiento español. Salud Colectiva, 10(3), 365-377.

Basu, Jayanti (2014). Psychologists' ambivalence toward ambiguity: Relocating the projective test debate for multiple interpretative hypotheses. SIS Journal of Projective Psychology \& Mental Health, 21(1), 25-36.

Billard, Thomas (2019). "Passing" and the politics of deception: Transgender bodies, cisgender aesthetics, and the policing of inconspicuous marginal identities. En Docan-Morgan, Tony (ed.). The Palgrave Handbook of deceptive communication (463-477). New York: Palgrave Macmillan.

Bullock, Wayne y Wood, Nicholas (2016). Psychological assessment with trans people. En Brabender, Virginia y Mihura, Joni (eds.). Gender and sexuality in psychological assessment (489-510). New York: Routledge.

Carroll, Lynne y Gilroy, Paula (2002). Transgender issues in counselor preparation. Counselor Education and Supervision, 41, 233-242.

Castro-Peraza, María Elisa, García-Acosta, Jesús Manuel, Delgado, Naira, Perdomo-Hernández, Ana María et al. (2019). Gender identity: The human right of depathologization. International Journal of Environmental Research and Public Health, 16(6), 978. doi: https://doi.org/10.3390/ijerph16060978

Coleman, Eli, Bockting, Walter, Botzer, Marsha, Cohen-Kettenis, Peggy, DeCuypere, Griet et al. (2012). Standards of Care for the health of transsexual, transgender, and gender non-conforming people (7th edition) of The World Professional Association for Transgender Health. International Journal of Transgenderism, 13(4), 165-232. doi: https://doi.org/10.1080/15532739.2011.700873

Davy, Zowie (2015). The DSM-5 and the politics of diagnosing transpeople. Archives of Sexual Behavior, 44, 1165-1176. doi: https://doi.org/10.1007/S10508-015-0573-6

de la Hermosa Lorenci, Marina (2018). Estrés de minoría y personas trans*: Herramientas para el acompañamiento afirmativo. En Cli- 
ment Clemente, M. ${ }^{a}$ Teresa y Carmona Osorio, Marta (eds.). Tranpsiquiatría: Abordajes queer en salud mental (77-99). Madrid: Asociación Española de Neuropsiquiatría.

Domino, George y Domino, Marla (2006). Psychological testing: An introduction. Cambridge: Cambridge University Press.

Drescher, Jack (2010). Queer diagnoses: Parallels and contrasts in the history of homosexuality, gender variance, and the diagnostic and statistical manual. Archives of Sexual Behavior, 39, 427-46o. doi: https://doi.org/10.1007/s10508-009-9531-5

Drescher, Jack, Cohen-Kettenis, Peggy y Winter, Sam (2012). «Minding the body»: Situating gender identity diagnoses in the ICD-11. International Review of Psychiatry, 24(6), 568-577. doi: https://doi.org/10.3109/09540261.2012.741575

Elischberger, Holger, Glazier, Jessica, Hill, Eric y Verduzco-Baker, Lynn (2018). Attitudes toward and beliefs about transgender youth: A cross-cultural comparison between the United States and India. Sex Roles, 78, 142-160. doi: https://doi.org/10.1007/s11199-017-0778-3

Esteva de Antonio, Isabel, Gómez-Gil, Esther y GIDSEEN Group (2013). Coordination of healthcare for transsexual persons: A multidisciplinary approach. Current Opinion in Endocrinology, Diabetes, and Obesity, 20, 1-7. doi: https://doi.org/10.1097/o1.med.oooo436182.42966.31

European Commission (2020). Legal gender recognition in the EU: the journeys of trans people towards full equality. Luxembourg: Publications Office of the European Union. Recuperado de: https://ec.europa.eu/info/sites/info/files/legal_gender_recognition_i n_the_eu_the_journeys_of_trans_people_towards_full_equality_sept _en.pdf

Francia-Martínez, Margarita, Esteban, Caleb y Lespier, Zahira (2017). Actitudes, conocimiento y distancia social de psicoterapeutas con la comunidad transgénero y transexual. Revista Puertorriqueña de Psicología, 28(1), 98-113. 
Fricker, Miranda y Jenkins, Katharine (2017). Epistemic injustice, ignorance, and trans experience. En Garry, Ann, Khader, Serene y Stone, Alison (eds.). The Routledge companion to feminist philosophy (268-278). New York: Routledge.

Geisinger, Kurt (1994). Cross-cultural normative assessment: Translation and adaptation issues influencing the normative interpretation of assessment instruments. Psychological Assessment, 6(4), 304-312. doi: https://doi.org/10.1037/1040-3590.6.4.304

Gómez-Gil, Esther, Esteva de Antonio, Isabel, Fernández Rodríguez, María, Almaraz Almaraz, Maricruz et al. (2020). Nuevos modelos de atención sanitaria para las personas transgénero en el sistema sanitario español: Demandas, controversias y reflexiones. Revista Española de Salud Pública, 94(16), 1-14.

Gregori Flor, Nuria, García Dauder, Dau y Hurtado, Inmaculada (2018). Bioethics and intersex: "time out". A paradigm shift on intesex management in the Spanish context. Athenea Digital, 18(2), e1899, 129. doi: https://doi.org/10.5565/rev/athenea.1899

Hambleton, Ronald y Kanjee, Anil (1995). Increasing the validity of cross-cultural assessments: Use of improved methods for test adaptations. European Journal of Psychological Assessment, 11(3), 147157. doi: https://doi.org/10.1027/1015-5759.11.3.147

Hines, Sally (2007). TransForming gender: Transgender practices of identity, intimacy and care. Bristol: The Polity Press.

Ho, Michelle (2021). Categories that bind: Transgender, crossdressing, and transnational sexualities in Tokyo. Sexualities, nn. pp. doi: https://doi.org/10.1177/13634607211028109

Hogan, Thomas (2015). Psychological testing: A practical introduction. Danvers, MA: Wiley \& Sons.

Jarrín, Álvaro (2016). Untranslatable subjects: Travesti access to public health care in Brazil. TSQ: Transgender Studies Quarterly, 3(3-4), 357375. doi: https://doi.org/10.1215/23289252-3545095 
Johnson, Austin (2016). Transnormativity: A new concept and its validation through documentary film about transgender men. Sociological Inquiry, 86(4), 465-491. doi: https://doi.org/10.1111/soin.12127

Kreukels, Baudewijntje, Hebold Haraldsen, Ira Ronit, Cuipere, Griet, Richter-Appelt, Hertha, Gijs, Luk y Cohen-Kettenis, Peggy T. (2010). A European network for the investigation of gender incongruence: The ENIGI initiative. European Psychiatry, 27, 445450. https://doi.org/10.1016/j.eurpsy.2010.04.009

Krishnamurthy, Radhika (2016). Gender considerations in self-report personality assessment interpretation. En Brabender, Virginia y Mihura, Joni (eds.). Gender and sexuality in psychological assessment (128-148). New York: Routledge.

Lev, Arlene Istar (2004). Transgender emergence: Therapeutic guidelines for working with gender-variant people and their families. New York: Haworth Clinical Practice Press.

López-Sáez, Miguel y García Dauder, Dau (2020). Los test de masculinidad/feminidad como tecnologías psicológicas de control de género. Athenea Digital, 20(2), e2521, 1-30. doi: https://doi.org/10.5565/rev/athenea.2521

López-Sáez, Miguel, García Dauder, Dau, Montero, Ignacio y Lecuona, Óscar (2021). Adaptation and Validation of the Evasive Attitudes of Sexual Orientation Scale into Spanish. Journal of Homosexuality, 69, 1-21. doi: https://doi.org/10.1080/00918369.2021.1898803

Martin, Hale y Finn, Stephen (2010). Masculinity and femininity in the MMPI-2 and MMPI-A. Minneapolis: Minnesota University Press.

Mason-Schrock, Douglas (1996). Transsexuals' narrative construction of the "true self". Social Psychology Quarterly, 59(3), 176-192.

Mason-Schrock, Douglas, Holden, Daphne y Reid, Lori (2004). Creating Emotional Resonance: Interpersonal Emotion Work and Motivational Framing in a Transgender Community. Social Problems, 51(1), 61-81.

Miach, Patricia, Berah, Ellen, Butcher, James y Rouse, Steve (2000). Utility of the MMPI-2 in assessing gender dysphoric patients. Journal of Personality Assessment, 75(2), 268-279. doi: https://doi.org/10.1207/S 15327752JPA7502_7 
Missé, Miquel (2018). A la conquista del cuerpo equivocado. Barcelona: Egales.

Monro, Surya (2007). Transmuting gender binaries: The theoretical challenge. Sociological Research Online, 12(1), 90-104. doi: https://doi.org/10.5153/sro.1514

Nichols, Margaret (2019). Supervision of therapists working with transgender clients. In Wadley, James y Siegel, Richard (eds.). The art of sex therapy supervision. New York: Routledge.

Páez, José, Hevia, Guillermo, Pesci, Florencia y Rabbia, Hugo (2015). Construcción y validación de una escala de actitudes negativas hacia personas trans. Revista de Psicología, 33(1), 151-188.

Paulson, Kesley, Straus, Elizabeth, Bull, Diana, MacArthur, Sarah, DeLorme, Jillian y Dalenberg, Constance (2019). Knowledge and views of psychological tests among psychiatrists and psychologists. Journal of Forensic Psychology Research and Practice, 19(2), 112-127. doi: https://doi.org/10.1080/24732850.2018.1546071

Richards, Christina, Bouman, Walter, Seal, Leighton, Barker, Meg John, Nieder, Timo y T'Sjoen, Guy (2016). Non-binary or genderqueer genders. International Review of Psychiatry, 28(1), 95-102. doi: https://doi.org/10.3109/09540261.2015.1106446

Riggle, Ellen, Scales, Sharon, McCants, LaWanda y Pascale-Hague, David (2011). The positive aspects of a transgender self-identification. Psychology and Sexuality, 2(2), 147-158. doi: https://doi.org/10.1080/19419899.2010.534490

San Emeterio, Azucena (2020). Psicoterapia feminista y gestalt. Revista de Terapia Gestalt, 40, 23-36.

Sennott, Shannon (2010). Gender disorder as gender oppression: A transfeminist approach to rethinking the pathologization of gender non-conformity. Women and Therapy, 34(1-2), 93-113. doi: https://doi.org/10.1080/o2703149.2010.532683

Shulman, Grant, Holt, Natalie, Hope, Debra, Mocarski, Richard, Eyer, Joshua y Woodruff, Nathan (2017). A review of contemporary assessment tools for use with transgender and gender nonconforming 
adults. Psychology of Sexual Orientation and Gender Diversity, 4(3), 304-313. doi: https://doi.org/10.1037/sgdoooo233

Singh, Anneliese y dickey, lore m. (2017). Introduction. En Singh, Anneliese y dickey, lore m. (eds.), Affirmative counseling and psychological practice with transgender and gender nonconforming clients (3-18). Washington, DC: American Psychological Association.

Slick, Daniel y Sherman, Elisabeth (2012). Differential diagnosis of malingering and related clinical presentations. En Sherman, Elisabeth y Brooks, Brian (eds.). Pediatric forensic neuropsychology (113135). New York: Oxford University Press.

Sue, Derald Wing (2010). Microaggressions in everyday life: Race, gender, and sexual orientation. New Jersey: Wiley \& Sons.

Suess Schwend, Amets (2020). Trans health care from a depathologization and human rights perspective. Public Health Reviews, 41(3), 1-17. doi: https://doi.org/10.1186/s40985-020-0118-y

Vidal-Ortiz, Salvador (2008). Transgender and Transsexual Studies: Sociology's influence and future steps. Sociology Compass, 2(2), 433450. doi: https://doi.org/10.1111/j.1751-9020.2007.00086.x

Wilkinson, Willy (2014). Cultural competency. TSQ: Transgender Studies Quarterly, 1(1-2), 68-73. doi: https://doi.org/10.1215/23289252-2399641

World Health Organization (2019). International statistical classification of diseases and related health problems (11th ed.). Recuperado de: https://icd.who.int/ 